1

\title{
DEL DISCURSO CIENTÍFICO \\ EN LO SOCIAL: \\ LA PUESTA EN DISCURSO \\ DEL CONOCIMIENTO
}

\author{
Carlos Alberto Caudana \\ Director del Proyecto
}

Desde el propio discurso, de su aparición y regularidad... [avanzar] hacia sus condiciones externas de posibilidad.

Michel Foucault

El orden del discurso 
La emergencia y constitución de un determinado dominio disciplinar se define, habitualmente, a partir de la construcción de un específico objeto de estudio y por sus relaciones con un marco pertinente de referencias teóricas y metodológicas. Sin embargo, al menos en el ámbito de las ciencias humanas y sociales, no sólo dicho objeto no se corresponde con actividades propias de la "exterioridad del hombre", sino que el mismo surge como proceso o resultado de su esencial "actividad de simbolización". Esto es: de todo aquello que adquiere sentido a través de la ejecución de sus acciones, de las interrelaciones que establece con su medio sociocultural $y$, fundamentalmente, de los discursos que produce.

A diferencia de los valores explicativos (más o menos verdaderos, únicos y universales) que caracterizan a otras ciencias, los principios constructivos que prevalecen en las disciplinas del campo humanístico y social no pretenden agotar la significación del objeto ni dar cuenta de su "totalidad". Son más bien "locales o circunstanciales", debido a los múltiples parámetros y perspectivas de abordaje que demandan para la captación de un determinado fenómeno (muy difícil de aislar y fragmentar, por otra parte).

Tampoco tales explicaciones procuran constituirse en leyes o principios "acumulativos", toda vez que sus enunciados proposicionales siempre referencian saberes y conocimientos de alguna (aunque relativa) validez. Que incluso pueden superponerse o solaparse con otros constructos teóricos o metodológicos, los que a menudo admiten ser revisados, corregidos, reactualizados, cuando no sustituidos...

Así, las prácticas de enseñanza e investigación aplicadas a determinados objetos, acontecimientos y procesos del dominio humanístico y social, la mayoría de las veces interactúan con argumentaciones, dilemas o controversias de índole teórica, epistémica, metodológica, etc., y casi nunca (por no decir nunca) con cosas, circunstancias y situaciones (más o menos) "objetivas". Al menos, tal como ellas "son" o se "aparecen", en la realidad-de-los-hechos... Cuando se las considera en "sí-mismas", quiero decir: sin sus correspondientes (inter)mediaciones... 
Reflexionar, entonces, acerca de las singularidades e improntas de la enseñanza y la investigación en humanidades y ciencias sociales (intentando compatibilizar indagaciones teóricas y observaciones efectuadas sobre referentes "concretos") supone concebir un planteamiento conceptual y metodológico de características heterogéneas, que procure resolver al mismo tiempo cuestiones relacionadas con la integración y transposición de diferentes saberes. Saberes inherentes, por un lado, a los desarrollos específicos de cada territorio disciplinar $y$, por otro, a las complejas intersemiosis que constituyen los objetos (también) específicos de su enseñanza e investigación.

En este contrapunto interseccionan y disputan a su vez otros conocimientos que, con frecuencia, alimentan las controversias suscitadas por la didáctica y la investigación social mismas, en tanto dominios que se construyen en la "encrucijada" de diversas disciplinas y en interacción "dialéctica" con sus respectivos marcos referenciales.

En síntesis, los conocimientos y saberes de nuestro campo científico se corresponden más bien con disciplinas de comprensión que de producción; rara vez permiten prever la "reproducción" de un fenómeno o la generación de un "producto" nuevo. En este sustancial aspecto de la cuestión se asienta el carácter inter(trans)disciplinario del dominio humanístico y social y sus correlativas aperturas a diversas filiaciones temáticas y a distintos tipos de cadenas explicativas.

Como dice Patrick Charaudeau', de lo que se trata es de abrir uno o varios caminos "entre el ostracismo disciplinar, en nombre de la cientificidad, y la ausencia de referencia disciplinar, en nombre de la complejidad". También es posible "construir una transversalidad entre varias disciplinas" pero "a condición de que se haga desde un lugar geométrico, [desde un nuevo] lugar disciplinar sin el cual ya no habría validación posible del saber" [2003: 19]. Coincidiendo con él la caracterizaré, asimismo, como práctica investigativa singular de una <interdisciplinariedad focalizada>.

${ }^{1}$ Charaudeau, P. (1997). Le discours d'information médiatique. París: Ed. Nathan. Cf. trad. cast. de M. Mizraji (2003). El discurso de la información: la construcción del espejo social. Barcelona: Gedisa. 


\section{DEL SENTIDO Y LO SOCIAL EN EL DISCURSO CIENTÍFICO}

La investigación acerca del sentido social, aplicado a través de la producción, circulación e interpretación de los signos que lo constituyen, con frecuencia suele complejizarse. Precisamente porque las indagaciones sobre "el sentido" ponen en juego ideas de pluralidad, de diversidad, de simultaneidad: referencias todas a las múltiples (semio)esferas ${ }^{2}$ en que vivimos y a los permanentes desplazamientos que (de una a otra) acometemos.

Es también por ello que las categorías teóricas y los medios y procedimientos utilizados para procurar investigarlo no pueden proceder sólo de circunscripciones monodisciplinares. Al mismo tiempo, abordar el estudio de los discursos sociales desde la perspectiva del sentido requiere no solamente instrumentos u operatorias analíticas para su descripción, sino también planteos hipotéticos sobre la "significación" social y acerca del "funcionamiento" de los objetos del (en el) campo (social). De modo tal de poder profundizar, además, en las dimensiones interpretativas y explicativas de los discursos en cuestión...

Por cierto: en este contexto, los enfoques semiodiscursivos y semionarrativos postulan la posibilidad de percibir y "narrar" los <objetos del mundo > a través de determinadas categorías del sentido, las cuales a su vez dependen de diferentes variabilidades de <la forma $>$.

Esta compleja construcción opera conforme a una dinámica metodológica de características basculares: hacia el exterior de los lenguajes, "referencia y representa" el mundo captado (empíricamente); hacia el interior, "identifica y formaliza" diferentes categorías de uso, las cuales dan cuenta de distintos procesos (constructivos) de selección, combinación, jerarquización, etc. Dicho de otro modo: las formas (de los signos) poseen autonomía significante, portan

\footnotetext{
2 Todo el espacio semiótico "puede ser considerado como un mecanismo único (si no como un organismo)". "La semiosfera es el espacio semiótico fuera del cual es imposible la existencia misma de la semiosis: [...] sólo la existencia de tal universo hace realidad el acto sígnico particular". Cf. Lotman, I. (1984). "Acerca de la semiosfera", en La semiosfera, I. Semiótica de la cultura y del texto [selec. Desiderio Navarro]. Madrid: Cátedra, $1^{\text {a }}$ ed. cast. (1996: 24).
} 
(sus propios) contenidos y significaciones ${ }^{3}$, dan cuenta de la semiotización del (de los objetos del) mundo...

Así, L.J. Prieto ${ }^{4}$ y M. Angenot ${ }^{5}$ conciben a la semiótica como "una ciencia histórica y social (aplicada a) las maneras de conocer y de representar lo conocido"; también sostienen que dichos modos de conocimiento y de representación están siempre "ligados a una función, a una praxis". En tal sentido, toda práctica (crítica) de lectura semiótica, transpolada a un determinado <hacer> específico del campo humanístico y social (lo lingüístico, lo historiográfico, lo espectacular, lo didáctico...), opera instituyendo sus propios saberes y conocimientos disciplinares, a la vez que re-constituye el mundo de sus <objetos> y a sus <sujetos> cognoscentes (esto es, a los productores y destinatarios de tales saberes) en tanto objetos de estudio del mismo dominio.

Pretender, en consecuencia, la reconstrucción (siquiera sea a través de mínimas evidencias fragmentarias) de cualquier forma o manifestación de discurso social, con el propósito además de analizar, comprender e interpretar aquello que (en la discursividad verbal, espectacular, audiovisiva, hipermedial, etc.) se codifica o decodifica, construye o deconstruye, se enuncia y se lee, se muestra o representa, se narra o argumenta..., se explicita, omite, sobrentiende..., se explana o se solapa..., demanda reformular un conjunto de problemáticas teóricas y metodológicas, todas ellas relacionadas con el ejercicio mismo de tales "prácticas discursivas".

\footnotetext{
${ }^{3}$ Al respecto sostuve, en una anterior oportunidad (vale ahora como ejemplo; cf. nota 10), que "las construcciones discursivas de carácter didáctico e investigativo no sólo podrían ser pensadas como estructuras funcionales, operatorias y transpositivas de distintos contenidos disciplinares específicos, sino también como un campo de estrategias discursivas que sostienen los sujetos (de dicho 'hacer'), cuyas interacciones también producen específicos 'contenidos' diferenciales (a menudo implícitos o sobrentendidos)". También, que "el reconocimiento y análisis de estos procedimientos formales, de tales articulaciones constructivas y deconstructivas del sentido y la significación, no sólo resisten la simplificadora interpretación de lo evidente, sino que permiten reflexionar sobre cuestiones que, en alguna medida, 'anteceden' al efectivo funcionamiento de las prácticas empíricas... Las cuales sí se sitúan en contextos explícitos, específicos: el aquí-y-ahora de las prácticas, a su vez 'localizadas' en determinados contextos institucionales" [Caudana, 2004: 11-2].

${ }^{4}$ Cf. Prieto, L. (1993). "Una semiología: problemas y resultados", en Estudios Nro. 2. Córdoba: Publicaciones del Centro de Estudios Avanzados/UNC.

${ }^{5}$ Cf. Angenot, M. (1998). Interdiscursividades. De hegemonías y disidencias. Córdoba: Editorial Universitaria.
} 
Con su correspondiente "gramática" del sentido (por ejemplo) y con sus propias reglas de producción, reconocimiento, circulación y consumo [E. Verón ${ }^{6}$ ]. Con la manifestación de sus diversas materialidades significantes [A.J. Greimas ${ }^{7}$; con sus específicas sustancias y formas diferenciales [L. Hjemslev ${ }^{8}$ ], en tanto aspectos del contenido y la expresión (estrategias, dispositivos, soportes, modalidades) de las referidas prácticas sociales...

\section{DEL DISCURSO CIENTÍFICO SOBRE EL SENTIDO Y LO SOCIAL}

Nuevos aportes y reformulaciones posformalistas a la (en un principio, denominada) semiótica textual de base lingüística han ido superando aquella primera instancia, meramente heurística, acerca de un cierto "saber" sobre los textos, en general orientado a la decodificación de sus materialidades significantes y a la aplicación de diversos procedimientos sistemáticos de descrip-

${ }^{6}$ Cf. Verón, E. espec. (1984). "Semiosis de lo ideológico y del poder", en Espacios Nro. 1. Buenos Aires: Fac. FyL/ UBA; (1985). La mediatización. Hacia una teoría de los discursos sociales. Buenos Aires: Fac. FyL/UBA; y (1987). La semiosis social. Fragmentos de una teoría de la discursividad. Barcelona: Gedisa.

${ }^{7}$ Cf. Greimas, A. J. espec. (1983). La semiótica del texto. Barcelona: Paidós; y (1989). Del sentido II. Ensayos semióticos. Madrid, Gredos. Asimismo, Greimas-Courtès (1982-91). Semiótica. Diccionario razonado de la teoría del lenguaje, I y II. Madrid: Gredos.

${ }^{8}$ En la concepción lingüística de L. Hjelmslev, retomada por el semiotista A.J. Greimas, cuando un código asocia elementos de un sistema transmisor con elementos de un sistema transmitido, el primero se convierte en la expresión del segundo, el cual, a su vez, se transforma en el contenido del primero. Existe "función semiótica" cuando una expresión y un contenido entran en correlación: ambos se convierten así en "funtivos" de tal correlación.

Cada uno de dichos planos del signo (la expresión y el contenido) admite a su vez ser entendido desde lo "formal" y lo "sustancial". Coincidiendo con Saussure en que la lengua es forma y no sustancia, Hjemslev postula que la "forma semiótica" es una invariante que da cuenta de la red relacional que define las unidades, y que la "sustancia semiótica" (materia o "sentido"), en cuanto soporte de la significación, es una variable. Es decir: una determinada "forma" (textual, discursiva, narrativa, por ej.) puede manifestarse a través de muchas y diversas "sustancias" (lo fónico, lo gráfico, lo gestual, lo sonoro, lo audiovisual...), pero no a la inversa. 
ción. Reconstituido el esencial carácter proyectivo, dinámico de la semiosis, de su permanente apertura hacia nuevos aspectos del "mecanismo" propio de los conocimientos humanísticos [I. Lotman, B. Uspenski] ], la semiótica del texto procura hoy el abordaje de diferentes formas y modalidades, soportes y dispositivos del razonamiento discursivo en el campo sociocultural.

Desde esta perspectiva reconceptualizadora de lo semiótico (a la vez entendido como hacer y como reflexión-sobre-el-hacer en el dominio de las prácticas sociales), nuestro Proyecto de Investigación ${ }^{10}$ se fundamenta en la posibilidad de redefinir, recrear y transponer, en más de un sentido, algunas de sus principales contribuciones teóricas y metodológicas.

Considerar, por ejemplo, a los objetos semiósicos de acuerdo con específicos modos de producción, recepción, circulación y consumo; reconocer estrategias (de seducción, persuasión, manipulación...) a través de las cuales se constituyen dichos mecanismos interactivos; articular las componentes formales y conceptuales del proceso de textualización, según determinados recorridos "generativos" del sentido; recontextualizar las respectivas configuraciones discursivas en orden a determinadas culturas de referencia; extender la "idea" de narratividad a toda actividad humana significante...

En el marco conceptual enunciado, el Proyecto "Travesías del sentido" se propone reconocer nuevas dimensiones y categorías de análisis narratológico

${ }^{9}$ Cf. I. Lotman y B. Uspenski (2000). "Sobre el mecanismo semiótico de la cultura", en La semiosfera III. Semiótica de las artes y de la cultura (pp. 168-193). Madrid: Cátedra.

${ }^{10}$ El Proyecto de investigación y desarrollo "Travesías del sentido / indagaciones narrativas" (2005-07), aprobado y subsidiado por la Secretaría de Ciencia y Técnica de la Universidad Nacional del Litoral, constituye la octava fase de una línea general de investigaciones semióticas que, con mi dirección, se viene desarrollando en el ámbito de la Facultad de Humanidades y Ciencias desde el segundo semestre de 1987. Dicho proyecto en ejecución interactúa con otros proyectos igualmente evaluados, en el marco del Programa CAI+D denominado "Lenguajes, discursos y semiosis en las prácticas sociales".

Algunos aspectos sustanciales del diseño de dicho proyecto (y sus articulaciones con las restantes etapas de la línea general de investigación en desarrollo) se incluyen en el primer número de esta serie. Cf. al respecto Caudana, C. (2004). Travesías del sentido / Indagaciones narrativas. Revista De signos y sentidos, n 1 . Santa Fe: UNL. 
para la descripción e interpretación de situaciones de enseñanza e investigación aplicadas a determinados objetos, acontecimientos o procesos del dominio humanístico y social. En ese contexto, dichas entidades y prácticas analíticas podrán ser entendidas como repertorios formalizados de constructos narrativos, o bien referidas o transpuestas al estudio semiodiscursivo de distintos corpus textuales de relatos verbales, audiovisivos e hipermediales: mediáticos, espectaculares, ficcionales, documentarios, divulgativos, sincréticos, fronterizos, etc.

Se articularán entonces, con doble anclaje en el diseño del proyecto, puestas (narrativas) en sentido (textual) y prácticas sociales correlativas, a fin de contribuir a una mejor explicación y comprensión del entramado de contenidos y formas significantes que constituyen las complejas semiosis en tratamiento. Así será posible, por ejemplo, entre otras operaciones analíticas aplicadas al corpus, abordar ciertas modalidades constructivas del "pensamiento narrativo" subyacente a los textos en cuestión (a sus estrategias referenciales y principales soportes o dispositivos característicos), que se conjuga con una diversidad de lenguajes y discursos, y recorre sus procesos interactivos de configuración, desciframiento y consumo.

En este territorio conceptual y metodológico de sesgo interdisciplinar ${ }^{11}$ (en que semiótica textual, didáctica crítica e investigación social armonizan e intercambian sus saberes y conocimientos específicos), la propuesta del proyecto y su eventual impacto presuponen que aquel reconocimiento de nuevas entidades analíticas e interpretativas permitirá reconceptualizar unas y otras prácticas singulares, indagarlas desde entornos diferentes de los habituales, establecer nuevos contextos de referencia para la reflexión y comprensión de los problemas existentes en uno u otro campo, y enriquecer así la mirada que a menudo se proyecta sobre cada uno de ellos desde los aportes elaborados en el otro.

${ }^{11}$ Reitero aquí mi adscripción al principio de "interdisciplinariedad focalizada" [Charaudeau, 2003: 19]. 


\section{A MODO DE PRESENTACIÓN DE ESTE "CUADERNO"}

En este volumen se anticipan algunos lineamientos iniciales sobre el diseño de cinco trayectos correspondientes al desarrollo de dicha propuesta de investigación; unos y otros se relacionan con distintos objetos de interés semiótico: espectáculos, documentales, entrevistas, hipermedios, metateorías... Desde sus singulares y específicas perspectivas de abordaje, cada uno de estos itinerarios del proyecto general procurará dar cuenta, explicar y comprender los entramados de sentidos, contenidos y formas significantes que constituyen (y se constituyen en) los complejos procesos de construcción discursiva y textual en que se transpone o transfigura el "pensamiento" narrativo.

En el capítulo siguiente, por ejemplo, se plantean algunas primeras hipótesis de trabajo acerca de las relaciones existentes y límites posibles entre narración y representación en las construcciones del discurso teatral. El trayecto indagará en aspectos problemáticos de la teorización acerca de la diégesis y la mímesis, desde los principales referentes clásicos (Platón, Aristóteles) hasta algunos de los diferentes aportes de la teoría teatral contemporánea (Brecht, Artaud...), así como estudiará los distintos niveles de intervención receptiva que operan en la constitución de los procesos de la puesta en escena espectacular ${ }^{12}$.

A continuación se proponen diversas cuestiones y perspectivas de abordaje inicial en relación con el < filme documental>, partiendo de su consideración como "específico" objeto textual emergente de un determinado "contexto" sociocultural. La progresiva construcción del corpus analítico e interpretativo (actualmente en su etapa preliminar) irá dando cuenta de las articulaciones posibles entre las formas del contenido y la expresión en el texto fílmico documental, de los distintos recursos y procedimientos que permiten reconstituir discursos/relatos acerca de determinados "acontecimientos" de la realidad social contemporánea ${ }^{13}$.

\footnotetext{
${ }^{12}$ En la actualidad participan en la ejecución de este trayecto Verónica Bucci, Carolina Cano, Valeria Kippes y Cielo Parodi, con la coordinación de Fabián Mónaco.

13 Intervienen en este tramo del desarrollo de la propuesta, que coordina Hugo Ramos: Graciela Martínez, Paula Zimmerman y Alberto Bartolini.
} 
En el cuarto capítulo de este "Cuaderno" se desarrollan lineamientos de base de un diseño investigativo transpuesto al análisis de un objeto textual de características singulares. El propósito central de las indagaciones previstas para el desarrollo de este itinerario radica en el interés por abordar las construcciones narrativas de (el género discursivo de) la entrevista: lo cual supone focalizar a la vez las condiciones de producción de un discurso específico, en relación con las particularidades del evento comunicativo, y los problemas teóricos y metodológicos que genera la configuración (textual) de las fuentes orales y su interpretación ${ }^{14}$.

El quinto artículo propone los fundamentos y tramos iniciales de una investigación referida a la codificación y decodificación de textos electrónicos multimediales, de naturaleza semiótica diversa, cuya convergencia e hibridación de lenguajes exigen nuevas competencias comunicativas, espectatoriales, cognitivas, etc. La compleja "gramaticalidad" de las transacciones narrativas (supuestas por el uso de nuevos entornos tecnológicos) demanda abordajes fundados en perspectivas multidisciplinares, así como el desarrollo de propuestas didácticas que den cuenta de diferentes paradigmas de producción y reconocimiento de los mensajes hipermediales ${ }^{15}$.

Finalmente, en el sexto capítulo, se plantean algunas primeras orientaciones y perspectivas eje de trabajo a propósito de la ejecución de una propuesta investigativa que anticipa también sus particulares perfiles. El trayecto en cuestión procurará indagar los cruces operantes entre pensamiento narrativo y discurso científico (y su puesta en acción en las prácticas didácticas), a partir de la discusión y reenfoque de ciertos tópicos polémicos existentes en la actualidad: las problemáticas de la narratividad y la referencialidad en el dominio de los modos de construcción y representación del conocimiento social ${ }^{16}$.

\footnotetext{
${ }^{14}$ El presente trayecto es conducido por Fabiana Alonso; colaboran con ella en el desenvolvimiento del mismo: Valeria Pini, Luis Escobar y Luciano Montenegro.

${ }^{15}$ Con la coordinación de Natalia Bas, participan del diseño y ejecución de las actividades del trayecto: Verónica Gorriti, Patricia Micheloud y Juan J. Gonnet.

${ }^{16}$ Actualmente intervienen en la discusión de este recorrido investigativo Juan J. Chizzini, Luis Quintana, Jorge Sartor y Bonfilio Zanazzi, coordinados por Aquiles Kobialka.
} 
El enfoque conceptual y metodológico, a partir del cual se esbozan a continuación los lineamientos iniciales (de los respectivos itinerarios del proyecto general), se relaciona con algunos debates actuales que sitúan la necesidad de repensar los fenómenos sociales y educativos desde nuevas ópticas de abordaje y con recursos técnicos diferentes de los habituales. La historia de las investigaciones desarrolladas en el campo se ha estructurado (muchas veces) sobre la base de ciertos dualismos y dicotomías que es necesario neutralizar o recomponer si se pretende una efectiva reconceptualización de los procedimientos constructivos del conocimiento que recupere lo empírico y lo teórico, y que reconsidere las condiciones contextuales dentro de las cuales operan los esquemas científicos.

En el mismo sentido, unas y otras formulaciones programáticas postulan o se fundan en una "idea" constructiva de relato, el cual es concebido como proceso generativo textual ${ }^{17}$ que sólo puede ser comprendido e interpretado si se lo acompaña en su "recorrido" y se lo aborda, con una "mirada" integradora, en su esencial triangularidad constitutiva: acontecer-narrado (diégesis o histo-

\footnotetext{
${ }^{17}$ Concebidas como entidades analíticas fundamentales para el desarrollo de esta investigación, las nociones de "texto", "narración" y "discurso", y su posible articulación en torno al "constructo relato", demandan permanentes redefiniciones tanto en sus alcances conceptuales como operacionales. El problema radica en que dichas categorías presentan acepciones diversas y ofrecen una multiplicidad de usos o empleos, no sólo en la comparación de los términos entre semiótica y didáctica (o de éstas con otros dominios disciplinares afines), sino aun dentro de un mismo campo.

Algunas de ellas, inclusive, suelen ser utilizadas como nociones intercambiables y, muchas veces, con similares alcances significativos; en otras oportunidades, con matices apenas diferenciales, o directamente con diferentes sentidos (cuando no de manera contradictoria o ambigua). Esta heterogeneidad léxico-semántica podría quizás ser adjudicada a las distintas concepciones subyacentes a cada categoría, las cuales "arrastran" la significación de las restantes, según procedan de determinado contexto teórico, epistemológico, etc.

En una publicación anterior efectúo un primer relevamiento acerca del estado de dicha cuestión. Cf. al respecto Caudana, C. (1998). Sobre textos y discursos en las construcciones del sentido. [Serie "Temas de Humanidades", 3]. Santa Fe: CESIL (FHUC, UNL).
} 
ria, contenido formalizado), enunciado-narrativo (relato, o discurso efectivamente producido) y acción-narrativa (acto de producción o enunciación) ${ }^{18}$.

Esto es: identificando no sólo los distintos aspectos (temas, acciones, personajes, etc.) de la historia-por-contar, sino también los diferentes códigos que se implican en su "funcionamiento", y estableciendo entre ellos las relaciones que evidencian o manifiestan la polifonía textual que soporta y constituye la estructura (productiva) del discurso narrativo...

Precisamente. Por las virtualidades teórico-metodológicas (improntas e implicitaciones) que insinúa esta singular conceptualización de <lo narrativo $>$... es el dominio de la semiótica textual el que quizás se referencie como espacio transversal y geométrico más adecuado para el ejercicio de aquella "interdisciplinariedad focalizada"... ${ }^{19}$.

Porque, en tanto teoría general de las significaciones, propone una perspectiva de abordaje de los "objetos textuales" suficientemente abarcadora, arti-

\footnotetext{
${ }^{18}$ En tanto proceso generativo del sentido, una narrativa sería entonces la "resultante" de la compleja intersección de diégesis, relato y narración: tres "niveles" interdependientes de manifestación de un mismo fenómeno (textual). Así, desde una perspectiva "gramatical (quiero decir: morfosintáctica) del sentido narrativo", y considerando a lo audiovisivo fílmico (por ejemplo) como sistema de producción significante, la diégesis referiría el contenido fílmico narrativo: esto es, el significado-base e hilo conductor del relato (hechos, acciones, lugares, tiempos, personajes... significativos; los "mostrados" por el filme... pero también los sugeridos, presupuestos, implícitos). El relato, por su parte, se identificaría con el discurso fílmico narrativo efectivamente producido; vale decir, el enunciado "material" y "re-presentativo" del filme, o bien la totalidad del conjunto significante: todo lo visible y audible (la historia contada -por la imagen- y el modo de contarla). La narración, finalmente, se asociaría con el "acto (mismo) de narrar" lo fílmico (esto es: la producción-recepción-circulación del filme) que produce o enuncia el relato, y que se inscribe en una determinada situación (contexto) de producción, subyacente al "texto" efectivamente narrado.

Sobre el particular, cf. Caudana, C. (2005). El signo textual narrativo [Serie "Documentos del Proyecto", Nro. 5], e Intertextos heterogéneos: historias, relatos, narrativas... Revista De signos y sentidos, n 5 . Santa Fe: UNL (en preparación).

${ }^{19} \mathrm{Cf}$. supra, referencias nota 1.
} 
culadora incluso de criterios de lectura fundados en otras pertinencias, que facilita incluso la descripción de dicho proceso constructivo, sin la pretensión de ofertarse como método hermenéutico (exclusivo o excluyente) ni tampoco como exegética interpretativa o como un acceso infalible para el comentario textual.

Quiero decir: una semiótica textual que se preocupa o interesa, más que por explicar lo que los textos significan, por comprender cómo éstos significan... Mediante qué mecanismos y estrategias (narrativas) se genera la "producción" (social) del sentido, y cómo puede ser éste "reconstruido" en la instancia (social) de recepción...

Cuestiones, todas, que ciertamente tienen que ver con "otros" modos y formas (quizás menos ortodoxos o convencionales) de concretarse (en el texto) el dis-currir del pensamiento narrativo ${ }^{20}$. Al menos, en ciertos y determinados "casos", obviamente resultantes de la observación e investigación aplicadas a otras tantas prácticas sociales. Como, por ejemplo, los que se caracterizan a continuación...

\footnotetext{
${ }^{20}$ Entre otros casos, a través del examen del "discurso" de las significaciones (o de las formas en que se constituyen los significados) en determinadas "actuaciones" de docentes e investigadores, por ejemplo: al configurar la perspectiva teórica o la construcción metodológica de una clase, o al diagramar posibles itinerarios reconstitutivos de los saberes o conocimientos disciplinares, o al establecer permanentes estrategias contractuales en el ámbito del aula... [Caudana, 2004: 57-8]. Esto es: en los procesos o prácticas significantes que se "realizan" (diré: se "narrativizan") en diversos "textos" en los cuales, reinstaurados sus respectivos contextos (didácticos, investigativos), se construyen e interactúan diferentes sujetos, creencias, ideologías...
} 